\title{
Sarcoma cardiaco con metástasis a encéfalo y miasis
}

\author{
*Carlos Miguel Rios-González ${ }^{1,2}$, Fabiana Griselda Verón Mellid ${ }^{2}$ \\ ${ }^{1}$ Universidad Nihon Gakko, Dirección de Investigación, Fernando de la Mora. Paraguay \\ ${ }^{2}$ Universidad Nacional de Caaguazú, Facultad de Ciencias Médicas, Cnel. Oviedo. Paraguay
}

Cómo referenciar este artículo/ How to reference this article:
Ríos-González CM, Verón Mellid FG. Sarcoma cardiaco con metástasis a encéfalo y miasis. Mem. Inst. Investig. Cienc. Salud. 2019; 17(3): 76-78

\section{R E S U M E N}

Los sarcomas cardíacos, son una entidad muy rara que a menudo son asintomáticos hasta que están avanzados, y aun así producen síntomas inespecíficos e imitan otras patologías. El pronóstico sombrío resulta de una invasión local extensa y / o metástasis a distancia en la presentación. Se presenta una paciente de sexo femenino, de 24 años de edad, proveniente del área rural de Paraguay, que fue diagnosticado con sarcoma cardiaco primario. Un año después de la intervención quirúrgica, la paciente regresa al servicio, donde se detecta metástasis a encéfalo, concomitantemente se aprecia miasis, por lo que es ingresada a la unidad de cuidados intensivos, para seguimiento. Se puede apreciar la falta total de seguimiento a esta paciente, por lo que ha ingresado en este estado al servicio, por lo que es fundamental la implementación de programas de seguimiento a estos pacientes para evitar complicaciones prevenibles.

Palabras clave: Hipodermosis, Miasis, Metástasis de la Neoplasia.

\section{Cardiac sarcoma with encephalon metastasis and myiasis.}

\begin{abstract}
A B S T R A C T
Cardiac sarcomas are a very rare entity that are often asymptomatic until they are advanced, and even so produce nonspecific symptoms and mimic other pathologies. The somber prognosis results from extensive local invasion and / or distant metastasis in the presentation. We present a 24-year female patient from the rural area of Paraguay, who was diagnosed with primary cardiac sarcoma. One year after the surgical intervention, the patient returns to the service, where metastasis to the brain is detected, concomitantly myiasis is seen, and therefore, she is admitted to the intensive care unit for follow-up.The total lack of follow-up to this patient can be appreciated, which is why she has entered the service in this state, so it is essential to implement follow-up programs for these patients to avoid preventable complications.
\end{abstract}

Keywords: Hypodermyiasis, Myiasis, Neoplasm Metastasis

\section{INTRODUCCIÓN}

En los adultos, aproximadamente una cuarta parte de los tumores cardíacos primarios son malignos y los sarcomas representan la histología más común ${ }^{(1)}$. Los sarcomas cardíacos primarios son tumores agresivos que generalmente no producen síntomas hasta que están localmente avanzados. Estas manifestaciones tardías son inespecíficas e incluyen disnea, dolor torácico, insuficiencia cardíaca congestiva secundaria a obstrucción del flujo sanguíneo y síntomas sistémicos ${ }^{(2)}$. La mayoría de los tumores cardíacos malignos son metastásicos, casi siempre sarcomas y con su origen primario en el pulmón ${ }^{(3,4)}$.

Los primarios son extremadamente raros, con diversidad histológica, se originan en las distintas estructuras cardíacas y tienen un comportamiento biológico muy agresivo ${ }^{(5)}$. Debido a lo infrecuente de estos tumores la experiencia individual de profesionales es muy

- Los autores no declaran conflictos de intereses

Fecha de recepción: julio 2019. Fecha de aceptación: agosto 2019

*Autor correspondiente: Carlos Miguel Ríos González. Universidad Nihon Gakko, Dirección de Investigación, Fernando de la Mora. Paraguay

Email: carlosmigue_rios@live.com 
escasa y los datos provienen de estudios basados en la actividad quirúrgica y necropsias. Actualmente la incidencia de tumores cardíacos primarios es incierta, la histología no está completamente definida, el tratamiento no es efectivo y el pronóstico universalmente pobre, sin embargo, se estima una incidencia de $0,001 \%$ a $0,03 \%$ en las series de autopsias, siendo los tumores cardíacos primarios entidades extremadamente raras.

De hecho, los tumores metastásicos en el corazón son 30 a 50 veces más comunes de acuerdo a algunos autores ${ }^{(6-8)}$. El paciente puede permanecer asintomático durante un largo período de tiempo y debutar con síntomas cuando la enfermedad ya se encuentra en un estado avanzado, en vista de ello se presenta el siguiente caso de un paciente con sarcoma cardiaco con metástasis a encéfalo y miasis.

\section{PRESENTACION DEL CASO}

Se presenta una paciente de sexo femenino, de 24 años de edad, proveniente del área rural de Paraguay, que había acudido al servicio en su comunidad por disnea a los esfuerzos físicos de moderada intensidad y que fue tratada con tratamiento antibiótico por posible sepsis respiratoria. No mejorando su condición clínica e incluso apareciendo edemas en miembros inferiores y tos nocturna improductiva fue ingresada y compensada clínicamente en un hospital de su localidad, posteriormente siendo remitida a nuestro servicio, donde se constató soplo mitral.

Con la radiografía de tórax se verificó un aumento en el índice cardiotorácico, con el ecocardiograma transtorácico se observaba gran masa tumoral en aurícula izquierda, el resultado anatomopatológico concluyó que se trataba de un sarcoma de células fusiformes auricular con metástasis multiplex, procediendo al acto quirúrgico, con la conducta correspondiente.

Un año después de la intervención quirúrgica, la paciente regresa al servicio, donde se detecta metástasis a encéfalo, concomitantemente se aprecia miasis, por lo que es ingresada a la unidad de cuidados intensivos, para seguimiento (Figura 1).
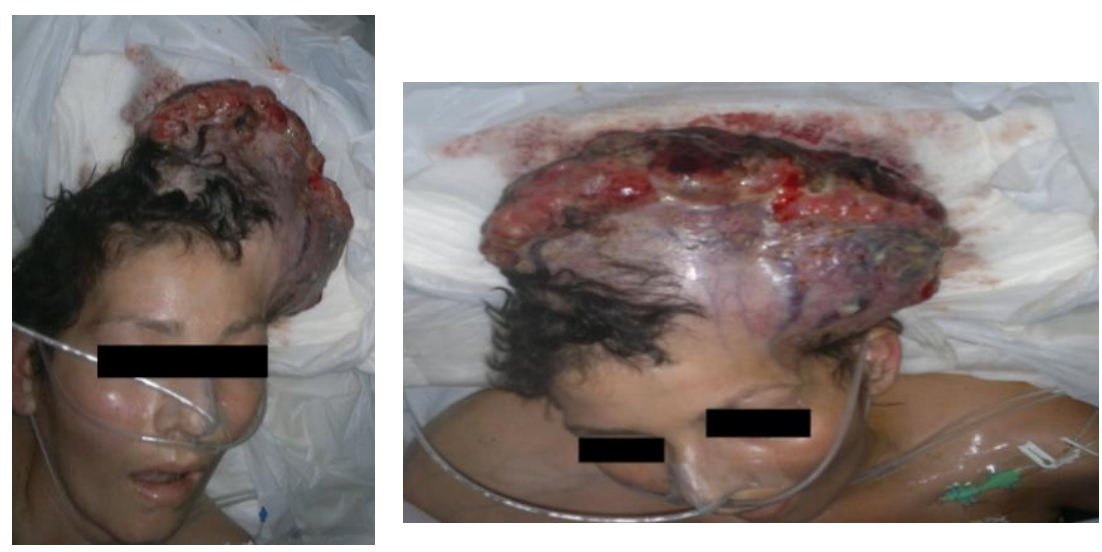

Figura 1: Un año después.

\section{DISCUSION}

Los sarcomas cardíacos son un subconjunto raro y distinto de sarcomas. Desafortunadamente, la mala supervivencia es la regla. La cirugía sigue siendo el tratamiento primario para el control local y la supervivencia en estos pacientes. Sin embargo, el uso de terapias adyuvantes se debe considerar sobre una base individualizada, particularmente en el contexto de enfermedades no resecables ${ }^{(9)}$.

El diagnóstico de los tumores cardíacos malignos se realizó debido a la aparición de síntomas de insuficiencia cardíaca congestiva que ocurre en más del $90 \%$ de estos pacientes ${ }^{(7,8)}$, con la ayuda indispensable de diversas técnicas de imagen ${ }^{(10)}$. La primera a utilizar en nuestro medio y a nivel internacional es la ecocardiografía transtorácica, seguida por la tomografía axial computarizada multicortes que permite, por su gran resolución temporal y espacial, una definición adecuada del tumor, a la cual no se pudo acceder en este caso, pero si bien se realizó la conducta adecuada para el caso, el seguimiento falló, puesto que después del alta, la paciente regresó un año después, ya con serios signos de mal cuidado en el hogar, si bien pudiera ser justificada en el contexto sociocultural precario en la cual se desenvuelvía ${ }^{(11)}$. 
En una búsqueda exhaustiva no se ha encontrado ningún caso similar, por lo que constituye el primer caso reportado tanto para la literatura paraguaya como para la literatura a nivel regional.

Por ello es fundamental que los hospitales cuenten con programas para seguimiento y control de este tipo de pacientes, evaluando detalladamente todo el contexto que rodea a las familias, de manera a reducir estas complicaciones prevenibles.

\section{REFERENCIAS BIBLIOGRAFICAS}

1. Kassop D, Donovan MS, Cheezum MK, Nguyen BT, Gambill NB, Blankstein R. et al. Cardiac Masses on Cardiac CT: A Review. Curr Cardiovasc Imaging Rep. 2014; 7:9281

2. Goldberg $A D$, Blankstein $R$, Padera RF. Tumors Metastatic to the Heart. Circulation. 2013; 128(16) : 1790-94.

3. Hoffmeier A, Sindermann JR, Scheld HH, Martens S. Cardiac tumors-diagnosis and surgical treatment. Dtsch Arztebl Int 2014; 111(12): 205-11.

4. Barreiro $M$, Renilla $A$, Jiménez JM, Martin M, Al Musa T, Garcia L et al. Primary cardiac tumors: 32 years of experience from a Spanish tertiary surgical center. Cardiovasc Pathol. 2013; 22(6): 424-7.

5. Díaz Angulo $C$, Méndez Díaz $C$, Rodríguez García E, Soler Fernández R, Rois Siso A, Marini Díaz M. Imaging findings in cardiac masses (Part I): study protocol and benign tumors. Radiologia 2015; 57(6):480-8.

6. Li X, Qi Q, Liang F, Zhang X, Dong S, Song B. Primary pulmonary artery sarcoma with deep vein thrombosis: A

case report. Medicine (Baltimore). 2019; 98 (23): e15874.

7. Vaitiekiene $A$, Vaitiekus $D$, Urbonaite $L$, Jankauskas A, Portacenko J, Lapinskas T, et al. Multidisciplinary approach to rare primary cardiac sarcoma: a case report and review. BMC Cancer. 2019; 19(1): 529.

8. Li X, Liu C, Zhang R. Primary cardiac malignant peripheral nerve sheath tumor: a case report. Int J Cardiovasc Imaging. 2019. 35(9):1615-18

9. Zhang G, Gao Q, Chen S, Chen Y. Primary cardiac synovial sarcoma that was continuous with the mitral valve caused severe thrombocytopenia: a case report. J Cardiothorac Surg. 2019; 14(1):30. doi: 10.1186/s13019-019-0852-8.

10. Skiba R, Stamp N, Kehoe M, Merry C. A rare pulmonary artery sarcoma, masquerading as pulmonary embolus. Ann Thorac Surg. 2019. pii: S00034975(19)30927-30. doi: 10.1016/j.athoracsur.2019.05.036.

11. Leonard MP. The importance of follow-up. Can Urol Assoc J. 2008; 2(1): 32. 